versals to the double lines. The residual section by the plane of $l, m, n$ is a conic whose image in the plane is also a conic, which has the common directions at $A$ and $B$ and passes through the four other points, thus fulfilling eight conditions. Thus, if four points of such a set of fourteen are collinear, or if eight of them lie in sets of four on two lines, the remaining points are related in the manner just noted. If we arranged the nine points in a triangle, one at each vertex and two on each side, we should get three skew lines on the surface, each meeting $l$ and four other lines, but not meeting the double lines.

The University of California

\title{
A NOTE ON THE RATIONAL PLANE QUARTIC CURVE WITH CUSPS OR UNDULATIONS*
}

BY J. H. NEELLEY

1. Introduction. In a recent paper $\dagger$ compound singularities of the rational plane quartic curve have been considered, but cusps and undulations were not incorporated in that article because of their widespread discussion by other writers. However, the errors or ambiguities in previous treatments of these two singularities are cleared up by this paper.

2. A System of Cusp Invariants. It is well known that all types of the rational plane quartic curve with simple singularities are given to within projection by plane sections of the Steiner Romische Surface $S_{3}^{4}$ of order three and class four. When referred to its tropes $S_{3}{ }^{4}$ has the equation

$$
\left(x_{0}\right)^{1 / 2} \pm\left(x_{1}\right)^{1 / 2} \pm\left(x_{2}\right)^{1 / 2} \pm\left(x_{3}\right)^{1 / 2}=0 .
$$

*Presented to the Society, December 28, 1927.

†J. H. Neelley, Compound singularities of the rational plane quartic curve, American Journal of Mathematics, vol. 49 (1927), pp. 389-400. 
If the intersection of the three double lines is taken as the unit point these lines have the equations

$$
\left\{\begin{array}{lll}
x_{0}=x_{1}, & x_{1}=x_{2}, & x_{2}=x_{0} \\
x_{2}=x_{3}, & x_{3}=x_{0}, & x_{1}=x_{3} .
\end{array}\right.
$$

The ends of the double lines are pinch points of the surface with the coordinates

$$
\begin{gathered}
(1,1,0,0),(1,0,1,0),(1,0,0,1),(0,1,1,0),(0,1,0,1) \text { and } \\
(0,0,1,1),
\end{gathered}
$$

and a plane on one of these points cuts out a curve with a cusp at the point. Let the coordinates of the cutting plane be $\left(\alpha_{0}, \alpha_{1}, \alpha_{2}, \alpha_{3}\right)$ so if it is on one of the pinch points, say $(0,1,1,0)$, we have $\alpha_{1}+\alpha_{2}=0$. As there are six pinch points there are six such forms each of which vanishes for some one of the pinch points. Therefore, one of the six factors of the product $\stackrel{6}{\Pi}\left(\alpha_{i}+\alpha_{j}\right)$ vanishes if the curve has a cusp. So this product may be used as an invariant which vanishes for a curve with a cusp. Letting $\sigma_{i}$ represent the symmetric functions of the $\alpha$ 's, this invariant may be written

$$
\sigma_{1} \sigma_{2} \sigma_{3}-\sigma_{3}{ }^{2}-\sigma_{1}{ }^{2} \sigma_{4}=0 .^{*}
$$

If we consider the curve with two cusps as cut out by a plane on two pinch points there are two forms of the type $\alpha_{i}+\alpha_{j}$ which vanish. So all possible products of five of the forms vanish and this gives as the two-cusp additional condition

$$
\sum^{6}\left[\stackrel{5}{\Pi}\left(\alpha_{i}+\alpha_{j}\right)\right]=0,
$$

which in terms of the symmetric functions is

$$
2 \sigma_{1} \sigma_{2}{ }^{2}+\sigma_{2} \sigma_{3}+\sigma_{1}^{2} \sigma_{3}-\sigma_{1} \sigma_{4}=0 . *
$$

But in so doing we neglect the fact that the plane cannot pass through both ends of one of the double lines. That is, a proper curve is cut out only if two forms such as $\alpha_{0}+\alpha_{1}$

*This invariant was given by J. E. Rowe, Transactions of this Society, vol. 12 (1911), p. 306. 
and $\alpha_{2}+\alpha_{3}$ do not both vanish. Imposing this condition on the $\alpha$ 's the two-cusp invariant is found to be

$$
\sigma_{1} \sigma_{3}+{\sigma_{2}}^{2}-4 \sigma_{4}=0 \text {. }
$$

Now let the plane $(\alpha x)=0$ be on three pinch points. Then three forms $\alpha_{i}+\alpha_{j}$ vanish simultaneously so that all products of four such forms vanish and we have

or

$$
\sum^{15}\left[\stackrel{4}{\Pi}\left(\alpha_{i}+\alpha_{j}\right)\right]=0,
$$

$$
\sigma_{1} \sigma_{3}+32 \sigma_{4}+\sigma_{2}^{2}+2 \sigma_{1}^{2} \sigma_{2}=0 .^{*}
$$

But again let us consider only proper curves and impose that no two forms such as $\alpha_{i}+\alpha_{j}$ and $\alpha_{k}+\alpha_{l}$ shall vanish simultaneously. This gives as the additional condition for a curve with three cusps the very simple invariant

$$
\sigma_{2}=0 \text {. }
$$

3. Degenerate Forms. Now we consider the possible degenerate forms cut from this surface. Such are either a conic and the square of a line or the square of a conic or the squares of two lines. The conic and the square of a line is gotten if the cutting plane passes through only two pinch points and these must be the ends of one of the double lines of the surface. That is, if $\alpha_{i}+\alpha_{j}$ vanishes $\alpha_{k}+\alpha_{l}$ vanishes also, whence $\sigma_{1}=0$ for such. And this is the condition that the plane pass through the unit point which is necessary for degenerate forms including a line. As two forms $\alpha_{i}+\alpha_{j}$ vanish simultaneously the condition (4) of the previous section is satisfied. So for a conic and line we have

$$
\sigma_{1}=\sigma_{3}=0 \text {, }
$$

as $\sigma_{2} \neq 0$, since the plane does not pass through three pinch points.

The square of a conic is cut from the surface if the plane passes through three pinch points which lie in one of the

* This invariant is given by $\mathrm{J}$. E. Rowe, loc. cit. 
reference planes. This means that condition (6) is satisfied and also $\sigma_{2}=\sigma_{3}=0$ due to condition (7) and the fact that the cutting plane is tangent to the surface. This gives $\sigma_{4}=0$ and so the square of a conic occurs if

$$
\sigma_{2}=\sigma_{3}=\sigma_{4}=0 \text {. }
$$

Now let the plane pass through two pinch points so that condition (5) is satisfied and also make $\sigma_{1}=0$ so that we have a pair of lines. This makes

$$
\sigma_{1}=\sigma_{2}=\sigma_{3}=\sigma_{4}=0,
$$

from consideration of relations (5) and (6).

So we have the following complete system:

A curve with one cusp has

$$
\begin{array}{ll}
\text { A curve with one cusp has } & \sigma_{1} \sigma_{2} \sigma_{3}-\sigma_{3}^{2}-\sigma_{1}^{2} \sigma_{4}=0 . \\
\text { Two cusps necessitates also that } & \sigma_{1} \sigma_{3}+\sigma_{2}^{2}-4 \sigma_{4}=0 . \\
\text { Three cusps adds to these } & \sigma_{2}=0 . \\
\text { A conic and square of } a \text { line if } & \sigma_{1}=\sigma_{3}=0 . \\
\text { The square of a conic if } & \sigma_{2}=\sigma_{3}=\sigma_{4}=0 . \\
\text { The squares of two lines if } & \sigma_{1}=\sigma_{2}=\sigma_{3}=\sigma_{4}=0 .
\end{array}
$$$$
\text { Three cusps adds to these }
$$$$
\text { The square of a conic if }
$$$$
\text { The squares of two lines if }
$$

4. The Curve with Undulations. There is another part of the literature of the rational plane quartic curve which should be revised. We refer to the theory of the curve with undulations as developed from consideration of the fundamental sextic which arises if the curve is represented parametrically. That is, if three forms

$$
x_{i}=\left(\alpha_{i} t^{4}, \quad(i=0,1,2),\right.
$$

represent the curve, the pencil of a polar quartics $(\beta t)^{4}+\lambda(\gamma t)^{4}$ fixes uniquely a sextic $(\rho t)^{6}$ which is apolar to the members of the pencil. The curve has been discussed by means of the invariants and covariants of this sextic.* Its line sections are the second polars of $(\rho t)^{6}$ and the pure second polars envelope the conic $K \dagger$ which is the locus of points in the plane from which a pencil of lines will cut out a pencil of

*W. Stahl, Über die rationale ebene Curve vierter Ordnung, Journal für Mathematik, vol. 104 (1889), pp. 302-320.

†W. Stahl, Über die rationale ebene Curve vierter Ordnung, Journal für Mathematik, vol. 101 (1886), pp. 300-325. 
binary quartics having the invariant $I_{2}$ zero. This conic $K$ breaks up into two lines when the quartic curve has an undulation; hence the statement of some writers that the sextic $(\rho t)^{6}$ is inadmissable when the curve has an undulation.* This statement is seemingly supported by the fact that the roots of $(\rho t)^{6}=0$ are the parameters of the points of the quartic curve where the lines of the curve and the conic $K$ at its corresponding points are incident. But let us examine the curve with an undulation. Calling the parameter of the undulation $t=0$ and that of a point of inflection $t=\infty$, the join of these two points and the lines of the curve at them gives the following representation of the curve:

$$
\left\{\begin{array}{l}
x_{0}=a_{0} t^{4}, \\
x_{1}=4 b_{1} t^{3}+6 c_{1} t^{2}+4 d_{1} t, \\
x_{2}=\quad 4 d_{2} t+e_{2},
\end{array}\right.
$$

whence the fundamental quartics are

$$
(\beta t)^{4} \equiv b_{1} t^{2}+c_{1} t, \quad(\gamma t)^{4} \equiv b_{1} d_{2} t^{4}+b_{1} e_{2} t^{3}-d_{1} e_{2} t^{2} .
$$

These quartics give

$$
(\rho t)^{6} \equiv K t^{6}
$$

which is very definitely the sixth power of the undulation parameter. This sextic (13) permits the recovery of but one line section and that is the undulation line of the curve. But this line is also a line of the degenerate conic $K$ whose equation is of the form

$$
x_{0}\left(K_{0} x_{0}+K_{1} x_{1}+K_{2} x_{2}\right)=0 .
$$

So at $t=0$, the point fixed by the root of $(\rho t)^{6}=0$, we have the line of the curve incident with a line of the conic $K$ even in this case. The unusual situation here is that the curve cannot be recovered from the sextic. This is generally possible

* W. Stahl, loc. cit., vol. 104, p. 303 and p. 318; R. M. Winger, Selfprojective rational curves of the fourth and fifth orders, American Journal of Mathematics, vol. 36 (1914), p. 64. 
as any three independent second polars of the sextic may be used as a reference scheme to recover the curve.

Consider a curve with undulations at $t=0$ and $t=\infty$. The join of the undulations and the undulation lines of the curve represent the curve as follows:

with

$$
\left\{\begin{array}{l}
x_{0}=a_{0} t^{4}, \\
x_{1}=4 b_{1} t^{3}+6 c_{1} t^{2}+4 d_{1} t, \\
x_{2}=\quad e_{2},
\end{array}\right.
$$

$$
(\beta t)^{4} \equiv b_{1} t^{2}+c_{1} t, \quad(\gamma t)^{4} \equiv b_{1} t^{3}-d_{1} t,
$$

which give

$$
(\rho t)^{6} \equiv \lambda t^{6}+\mu \tau^{4},
$$

or a pencil of sextics which includes the sixth powers of each undulation parameter. All second polars of (17) are lines of the pencil determined by the undulation lines of the curve and once more the curve cannot be recovered from the sextic as there are not three independent second polars. The conic $K$ for this type curve has the equation

$$
c x_{0} x_{2}=0,
$$

which is the product of the undulation lines, the two possible pure second polars of (17).

The curve with three undulations is well known but to make this article complete and to remove at least one erroneous statement concerning it we will give its theory. Let $t=0, \infty$ and $c_{1} / b_{1}$, where neither $c_{1}$ nor $b_{1}$ are zero, be the undulation parameters and use the undulation lines as a triangle of reference. Then the curve is given by

with

$$
\left\{\begin{array}{l}
x_{0}=a_{0} t^{4}, \\
x_{1}=\left(b_{1} t-c_{1}\right)^{4}, \\
x_{2}=e_{2},
\end{array}\right.
$$

$$
(\beta t)^{4} \equiv b_{1}^{2} t^{3}-c_{1}^{2} t, \quad(\gamma t)^{4} \equiv b_{1} t^{2}-c_{1} t
$$


which have the net of apolar sextics

$$
\lambda t^{6}+\mu\left(b_{1} t-c_{1} \tau\right)^{6}+\nu \tau^{6} .
$$

This net includes the sixth powers of the three undulation parameters as members. So the curve may be recovered from any member of the net (21) which is a linear combination of each of these sixth powers. This is because there is a net of second polars of such a member and any three independent ones may be used to recover the curve. The equation of the conic $K$ vanishes identically if the curve has three undulations. This is obviously necessary from the definition of $K$. That is, the pencil of lines determined by any two lines $(\lambda x)=0,(\mu x)=0$ cuts a pencil of quartics from the curve (19) for which the invariant $I_{2}$ vanishes. So the equation of $K$ must be satisfied by any point $\left(x_{0} x_{1} x_{2}\right)$ of the plane. We state the results of this section as the following theorems.

THEOREM 1. A curve with one undulation has as its fundamental sextic the sixth power of the undulation parameter and the curve cannot be recovered from the sextic.

THEOREM 2. A curve with two undulations has a pencil of fundamental sextics which includes the sixth powers of the undulation parameters and again the curve cannot be recovered from the sextics.

Theorem 3. A curve with three undulations has a net of fundamental sextics which includes the sixth powers of the undulation parameters and the curve may be recovered from any member of the net which is a linear combination of all three sixth powers.

Carnegie Institute of Technology 